\title{
CORRECTION TO "PRIMARY SOLUTIONS OF GENERAL BELTRAMI EQUATIONS"
}

\author{
Bogdan Bojarski \\ Instytut Matematyczny Polskiej Akademii Nauk \\ ul. Sniadeckich 8, 00-956 Warszawa, Poland; B.Bojarski@impan.gov.pl
}

As was noticed by several readers, I was not sufficiently careful in the formulation and the proof of Proposition 2 in [6]. The correct Beltrami equation for the function $G(\zeta)$-in the notation of [6], used throughout in this correction - is more complicated than stated in [6] and the use of its correct, in particular, quasilinear, form in a proof of the Theorem in [6] should be reconsidered. However the main Theorem as stated and the factorisation strategy for the proof proposed is valid.

Since the problem of "primary" solutions for Beltrami equations, started in the present form by Iwaniec et al. [7], aroused a rather long list of publications, extensively referred to, in particular, in the recent monograph [1], it seems proper to reconsider the topic from another perspective. The corresponding paper is in preparation.

Below we sketch another approach to the main theorem in [6]. The theorem reads as follows.

Theorem. The Beltrami equation

$$
\frac{\partial w}{\partial \bar{z}}-q(z)\left(\frac{\partial w}{\partial z}-\frac{\overline{\partial w}}{\partial z}\right)=0
$$

with the compactly supported measurable dilatation $(q \equiv 0$ in the neighbourhood of $\infty$ ) satisfying the ellipticity condition

$$
|q(z)|<k_{0}<\frac{1}{2}, \quad 2 k_{0}=k
$$

admits a global solution $\psi: \mathbf{C} \rightarrow \mathbf{C}$ satisfying condition

$$
\operatorname{Im} \frac{\partial \psi}{\partial z} \neq 0 \quad \text { a.e. }
$$

Then the pair $(z, \psi(z))$ is a primary pair for the Beltrami equation (1).

As is well known, see e.g. [8] and [5], the global homeomorphic solutions of (1), $w=\psi(z), \psi(\infty)=\infty$, admit the (unique) representation

$$
w(z)=a z-\frac{1}{\pi} \iint_{C} \frac{\omega(t) d \sigma_{t}}{t-z}, \quad a \neq 0,
$$

for some complex $a$ and $\omega \in L^{p}(\mathbf{C})$, where the density $\omega$ satisfies some uniquely solvable integral equation. It is convenient here to expose explicitly the dependence of the solution on the dilatation $q(z)$ and the coefficient $a$ interpreted as $\psi_{z}(\infty)=a$.

doi:10.5186/aasfm.2010.3521

2000 Mathematics Subject Classification: Primary 31C45, 30C65, 30C62.

Key words: Elliptic p.d.e., quasiconformal mappings, Beltrami equations, Lavrentiev characteristics, measurable Riemann mapping theorem. 
Thus we write $\psi(z) \equiv \psi(a, q, z)$. If $\operatorname{Im} a=0$, then the density $\omega \equiv 0$ and the solution (4) reduces to $w(z) \equiv a z$. If

$$
\operatorname{Im} a \neq 0,
$$

the solution $\psi=\psi(a, q, z)$ satisfies the requirements of the theorem.

The proof of the theorem for the "clasical" case when the dilatation $q(z)$ is smooth, say of class $C_{0}^{k}, k \geq 2$, follows immediately. Indeed, then the generalized solutions of (1) are smooth, e.g. Vekua [8] or any monographic treatment of elliptic 2D equations.

In particular, by the argument principle any complex (locally) homeomorphic solutions $w=w(z)$ of such equations do not allow any critical points, i.e.,

$$
d w\left(z_{0}\right) \neq 0, \quad w_{z}\left(z_{0}\right) \neq 0,
$$

for any finite $z_{0} \in \mathbf{C}$. Now by the quoted Vekua's and my works $[8,6]$ the global homeomorphic solutions of (general) Beltrami equations - the principal solutionsare classified by the coefficient $a \neq 0$ in (4). If, for a smooth dilatation $q(z)$, the solution $\psi \equiv \psi(i, q, z)$ would violate the condition (3) at a finite point $z_{0} \in \mathbf{C}$ then obviously some real combination

$$
w(z)=\alpha z+\beta \psi(z), \quad \alpha^{2}+\beta^{2}>0,
$$

for some $\alpha, \beta$ would produce a global homeomorphic solution of (1) with $a=\alpha+\beta a \neq$ 0 and a critical point at $z=z_{0}$, which is absurd. Thus for $q$ smooth the solution (4) satisfies the stronger inequality

$$
\operatorname{Im} a \cdot \operatorname{Im} \frac{\partial \psi}{\partial z}(a, q, z)>0
$$

everywhere.

For $q$ not smooth the solution $\psi(a, q, z)$ is the strong limit in $W_{\text {loc }}^{1, p}(\mathbf{C})$ (this means that the corresponding densities $\omega_{n} \rightarrow \omega$ in $L_{p}$ ) of smooth quasiconformal homeomorphisms $\psi\left(a, q_{n}, z\right), q_{n}(z) \rightarrow q(z)$ pointwise, by the convergence results for solutions of general Beltrami equations with measurable coefficients, discussed in detail in [6]. In consequence the inequality (3) follows in a weaker form

$$
\operatorname{Im} \frac{\partial \psi}{\partial z} \geq 0 \quad(\leq 0) \quad \text { for } \operatorname{Im} a>0(\operatorname{Im} a<0) .
$$

What is left to be shown is to exclude the possibility

$$
\text { mes } \Sigma_{\psi}>0, \quad \text { where } \Sigma_{\psi}=\left\{z: \operatorname{Im} \psi_{z}(a, q, z)=0\right\}
$$

if (5) is satisfied.

As we know now, there are several ways to achieve this result. They all work by reducing ad absurdum the assumption mes $\Sigma_{\psi}>0$. At a density point of the set $\Sigma_{\psi}$ the contradiction is obtained with a Belinskii-Wittich or Sard type theorem for homeomorphic quasiconformal mappings [3], by considering the family of QChomeomorphisms of $\mathbf{C}$, parametrized as follows:

$$
w(z)=\alpha z+\beta \psi(i, q, z)+w_{0}
$$

for $\alpha, \beta$ real and $w_{0}$ complex.

Also integral inverse Hölder inequalities can be used ([1], [2]). 


\section{References}

[1] Astala, K., T. Iwaniec, and G. Martin: Elliptic partial differential equations and quasiconformal mappings in the plane. - Princeton Math. Series 48, Princeton Univ. Press, 2009.

[2] Astala, K., and J. JäÄskeläinen: Homeomorphic solutions to reduced Beltrami equations. - Ann. Acad. Sci. Fenn. Math. 34, 2009, 607-613.

[3] Belinskil̆, P. P.: General properties of quasiconformal mappings. - Nauka, Novosibirsk, 1974 (in Russian).

[4] Bojarski, B.: Homeomorphic solutions of the Beltrami systems. - Dokl. Akad. Nauk SSSR (N.S.) 102, 1955, 661-664 (in Russian).

[5] Bojarski, B.: Generalized solutions of a system of differential equations of first order and of elliptic type with discontinuous coefficients. - Mat. Sb. (N.S.) 43:85, 1957, 451-503 (in Russian); English transl. in Rep. Univ. Jyväskylä Dept. Math. Stat. 118, 2009.

[6] Bojarski, B.: Primary solutions of general Beltrami equations. - Ann. Acad. Sci. Fenn. Math. $32,2007,549-557$.

[7] Giannetti, F., T. Inaniec, L. Kovalev, G. Moscariello, and C. Sbordone: On Gcompactness of the Beltrami equations. - In: Nonlinear Homogenization and its Applications to Composites, Polycrystals and Smart Materials, Kluwer Academie, Norwell, 2004, 107-138.

[8] Vekua, I. N.: Obobshchennye analiticheskie funktsii. - Fizmatgiz, Moscow, 1959.

Received 29 January 2010 